\title{
Meta-análisis: La reducción del colesterol con estatinas disminuye el riesgo de ACV y la mortalidad general
}

Cholesterol lowering with statin drugs, risk of stroke, and total mortality. Hebert P, Gaziano J, Chan K et al. JAMA 1997; 278: $313-321$

\section{Objetivo}

Meta-análisis para determinar si el descenso de los niveles de colesterol con estatinas disminuye el riesgo de ACV y la mortalidad general.

Fuentes de Datos

Revisión de la literatura para identificar estudios con inhibidos de HMG-CoA reductasa (estatinas) desde 1985 a 1995.

\section{Selección de Estudios}

Se seleccionaron 16 estudios randomizados en los que se

\begin{tabular}{|c|c|c|c|}
\hline $11=28701$ & $\mathrm{Acl}$ & 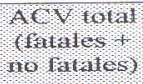 & $\begin{array}{l}\text { Macries } \\
\text { por todit } \\
\text { causa }\end{array}$ \\
\hline FST I IIIN $1 \mathrm{~S}$ & 31 & 193 & 532 \\
\hline$P 1+A(1, P O$ & 26 & 261 & 643 \\
\hline II ${ }^{2}, 5 \%$ & $\begin{array}{c}1.17 \\
0.69-1.97\end{array}$ & $\begin{array}{c}0.71 * * \\
0.59-0.86\end{array}$ & $\begin{array}{c}0.78 * * \\
0.69-0.88\end{array}$ \\
\hline riner & $17 \%$ & $29 \% * *$ & $22 \% * *$ \\
\hline
\end{tabular}

utilizaron estatinas como monodrogas y se reportaron datos sobre ACV y/o mortalidad. Se incluyeron tanto ensayos de prevención primaria de enfermedad coronaria como de prevención secundaria.

\section{Resultados Principales}

Las colesterolemias basales medias de cada estudio oscilaron entre 203 y $296 \mathrm{mg} \%$. Las reducciones medias fueron del 17 al 32 $\%$ para la colesterolemia y del 26 al $45 \%$ para LDL. Estas diferencias no fueron significativas entre las diferentes drogas evaluadas después de ajustar por distintas variables (edad promedio, $\%$ de mujeres, colesterolemia y LDL basales, y tipo de estudio prevención primaria vs. secundaria-). Se reportaron 454 ACV y 1.175 muertes entre casi 29.000 pacientes, a lo largo de 3.3 años de seguimiento promedio.

\begin{tabular}{|c|c|c|}
\hline$n=28701$ & 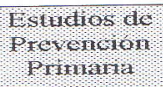 & 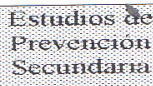 \\
\hline  & $20 \%$ & $32 \% * *$ \\
\hline Ket 950.0 & $\begin{array}{c}0.80 \\
0.54-1.16\end{array}$ & $\begin{array}{c}0.68 * * \\
0.55-0.85\end{array}$ \\
\hline Misurninicion & $26 \%$ & $21 \%$ \\
\hline $1 \mathrm{~T}^{\mathrm{N}}, 95 \%$ & $\begin{array}{c}0.74 \\
0.58-0.95\end{array}$ & $\begin{array}{c}0.79 \\
0.69-0.91\end{array}$ \\
\hline
\end{tabular}

De las 1.175 muertes totales, 796 fueron por causa cardiovascular, 373 no cardiovasculares (269 por cáncer) y 6 no categorizadas.

\begin{tabular}{|c|c|c|c|c|c|}
\hline $17=28,91$ & Mrortes $101 \mathrm{les}$ & Mineries & NoOCandionirsce & Grousde & 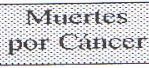 \\
\hline 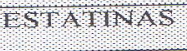 & 532 & 349 & 180 & 504 & 131 \\
\hline MIACLUY & 643 & 447 & 193 & 446 & 138 \\
\hline $11 \mathrm{r}, 0,0$ & $\begin{array}{c}0.78 * * \\
0.69-0.88 \\
\end{array}$ & $\begin{array}{c}0.72 * * \\
0.63-0.84\end{array}$ & $\begin{array}{c}0.93 \\
0.75-1.14\end{array}$ & $\begin{array}{c}1.03 \\
0.90-1.17\end{array}$ & $\begin{array}{c}0.95 \\
0.74-1.21\end{array}$ \\
\hline 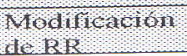 & $\downarrow 22 \% * *$ & $\downarrow 28 \% * *$ & $\downarrow 7 \%$ & $\uparrow 3 \%$ & $\downarrow 5 \%$ \\
\hline
\end{tabular}

Conclusiones

El descenso de los niveles del colesterol con estatinas dismi- nuye el riesgo de $\mathrm{ACV}$, especialmente en estudios de prevención secundaria, y disminuye la mortalidad general en todos los casos. tiva disminución del riesgo de infarto agudo de miocardio (a su vez factor de riesgo para $\mathrm{ACV}$ ) dado que el mayor beneficio se observa en la población previamente coronaria. En definitiva; cuando una intervención es probadamente efectiva sobre un evento importante como la coronariopatía y disminuye la mortalidad, solo debería probarse que no incremente el riesgo de otro evento peligroso, y no exigir que también lo disminuya. Podríamos entonces sacar las siguientes conclusiones: 1) Pacientes coronarios con hiperlipemia: las estatinas disminuyen el riesgo de eventos coronarios subsiguientes, el riesgo de ACV y la mortalidad general. Intervención hasta el momento indiscutible. 2) Pacientes coronarios sin hiperlipemia: también se benefician con la intervención (6). 3) Pacientes no coronarios hiperlipidémicos: el beneficio sobre el riesgo coronario ha sido demostrado (7) y sobre la incidencia de ACV no se han demostrado efectos perjudiciales. Por lo tanto la intervención es recomendable teniendo en cuenta factores de riesgo adicionales, principalmente coronarios. Para precisar el beneficio potencial sobre el riesgo de ACV sería interesante el desarrollo de estudios de prevención primaria y secundaria de enfermedad cerebrovascular.

* Ver glosario

Dr Alejandro Regueiro Sistemas de Protección Médica

\section{Referencias}

1. Prospective Studies Collaboration. Cholesterol, diastolic blood pressure, and stroke: 13000 strokes in

450000 people in 45 prospective cohorts. Lancet. 1995; 346: 1647-1653 2. Atkins D, Psaty BM, Koepsell TD et al. Cholesterol reduction and the risk for stroke in men.

Ann Intern Med. 1993: 119: 136-145

3. Holme I. Cholesterol reduction and its impact on coronary artery disease and total mortality.

Am J Cardiol. 1995; 76: 10C-17C.

4. Byington RP, Jukema JW, Salonen JT, et al. Reduction in cardiovascular events during pravastatin

therapy. Circulation. 1995; 92: 2419-2425.

5. Reed D, Yano K, Kagan A. Lipids and lipoproteins as predictors of coronary heart disease, stroke, and

cancer in the Honolulu Heart Program. Am J Med. 1986; 80: 871-878. 6. Sacks FM, Pfeffer MA, Moye LA et al. for the cholesterol and recurrent events trial investigators. The effect of pravastatin on coronary events after myocardial infarctions in patients with average cholesterol levels. N Engl J Med 1995; 332:488-93.

7. The west of Scotland coronary prevention study group. Prevention of coronary heart disease with pravastatin in men with hypercholesterolemia. N Eng1 J Med 1995,; 333:1301-07. 